\title{
Secured Cloud Resources Scheme Using Multi-Process Technique
}

\author{
N. Divya \\ M.Tech , Department of CSE, JNTUA College of Engineering, Ananthapuramu, Andhra Pradesh, India
}

\begin{abstract}
Article Info

Volume 7, Issue 1

Page Number: 155-160

Publication Issue :

January-February-2021

\section{Article History}

Accepted : 01 Feb 2021

Published : 08 Feb 2021

Cloud computing is a variety of service provider through the internet mobile cloud computing is a simplicity cloud computing in which same mobile devices included among them. As the mobile devices increasing in large number day by day. In existing system Mobile cloud computing used to overcome the limitations of smart phones such as battery life time, limited computational power, size of memory and uncertain network connectivity. To overcome this limitations in offloading technique novel frame work will be used in this files from user are transmitted to cloud in single path. This is more time consuming and takes more time in transmitting files. Proposed system we use this technique in edge cloud computing server to reduce latency and also to reduce energy consumption and perform task with efficiency. In addition parallelization is used for the execution of the method in cloud server to reduce energy and execution time.

Keywords : Cloud Computing, File and Parallel Transmission.
\end{abstract}

\section{INTRODUCTION}

Cloud Computing provides us many services through the internet where we can access the application as utilities. Mobile cloud computing is a framework where all the mobile users use the cloud servers and perform there tasks through the internet by connecting to the cloud. The mobile users are lack of resources like storage, efficiency and cpu utilization. Cloud provides resources to the mobile users without any maintenance it can provide resources like storage, execution, transmission of files.

Mobile cloud computing is a technique in which all the smartphone users perform there task through the cloud server as now a days smartphone devices are increase in limitations are also increasing we use some techniques to overcome this limitations such as battery lifetime and energy. Mobile users perform so many tasks by cloud computing such as data uploading, data mining etc. while perform this operations users are facing so many security issues the security threats have became obstacle in the rapid enhancement of data and large use of mobile computing paradigm we provide so many security techniques for the mobile users.

Smartphones are very useful for humans now a days they are used at every where for performing number of operations hence technology developing the usage of mobiles is also increasing but the mobiles are lot of limitations and drawbacks like battery draining, storage, consuming energy. In order to overcome these limitations we develop so many techniques like parallelization, optimization etc... considering one among the limitation energy consumption, we can

Copyright: (C) the author(s), publisher and licensee Technoscience Academy. This is an open-access article distributed under the terms of the Creative Commons Attribution Non-Commercial License, which permits unrestricted noncommercial use, distribution, and reproduction in any medium, provided the original work is properly cited 
make mobiles to perform the operation with less energy consumption in many ways, we can identify the power consumption application running in the background and try to optimize them to save the battery.

The main technique is used to overcome all the limitations is to provide services and resources to the mobile users over the internet and offload all the task remotely through the network

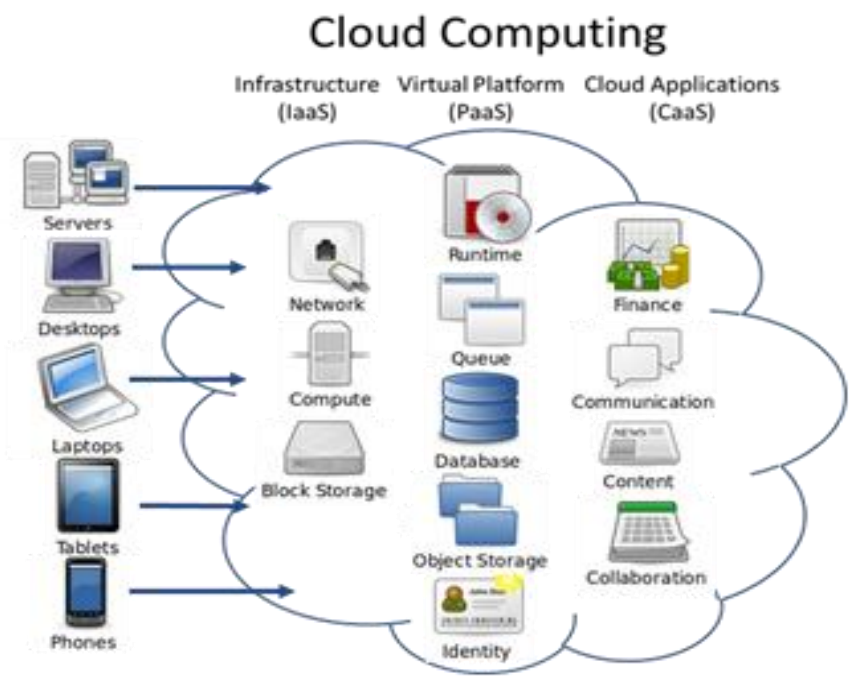

Figure 1 : Cloud Computing Architecture

In recent work cloud computing provide so many techniques for offloading mobile applications to the cloud from mobile devices for execution remotely these techniques are developed for the mobile devices to offload task with great efficiency including some constraints such as mobile as a consumption, cpu utilization, time of execution, battery lifetime and transmission of data. now a days the mobile users are facing big problem that is usage of memory. The memory is one of the resource consumed by the mobile apps and users. In addition to that security techniques are not used for the protection of data from attack while offloading the future technique focus on developing a new technique which combines the above constrain.
We use parallelization technique for transmission of data while offloading task as mobile users perform so many tasks at a time we can offload the task of mobile application while using the parallelization.

\section{RELATED WORK}

Large number of users access cloud to utilize services depending on there constrain. user's data should be secure and privately managed. Only authorized user have rights to access authorized data. This framework can only download methods of application that consume substantial mobile resources. As the current cloud server data transmission does not parallel transmission which is consuming a lot of transmission time and cost and also the current framework does not provides security layer which in the protect the data methods before transferring to the cloud. These existing techniques all require centralized control, time-honored adoption by all community nodes, or both; thus none of these strategies could offer incremental deployment of throughput most fulfilling routing to cloud networks. Moreover, those techniques cannot be used alongside throughput foremost dynamic manipulate schemes, such as backpressure.[4]

In cloud computing the task offloading can consume more energy and also takes more time now a days this is the big limitation in cloud computing to overcome this the authors Sokol Kosta and Anrius Auina developed a technique the technique involves multiple VM's to execute the task on the cloud on demand of the user it achieves mainly less execution time and energy consumption. They developed the technique by involving of Think Air components on the cloud which runs the task on commercial cloud .by using parallelizable applications and VMs the cloud can performs all the operations within the time and consuming the less energy had a framework that in which developers can easily drift their smart phones application for the cloud. Think Air extends 
the concept of virtualization among smart phones in the clouds and this can provide a method-level offloading computation. [1]

It proposes thematic taxonomy of current DAPFs, reviews current offloading frameworks by using thematic taxonomy and analyzes the implications and critical aspects of current offloading framework. Further, it investigates commonalities and deviations in such frameworks on the basis significant parameters such as offloading scope, migration granularity, partitioning approach, and migration pattern. Finally, we put forward open research issues in distributed application processing for MCC that remain to be addressed.in MCC. The mobile devices are connected to cloud through the network as mobile devices have less sufficient storage to store the large amount of data and less energy to perform the operations so that the mobile devices are connected to the cloud structure basing on their attribute and perform all the operations on a demand the cloud provide the resources such as security, storage, task execution etc. all the mobile applications cannot perform the user operations because they lack with insufficient storage and less energy. In MCC all the mobile users connected to cloud as per their need they perform the task through the cloud. As mobile devices are moving for one place to another place they are connected to nearest cellular network and use the cloud resource. The cloud manager scheduling the task given by the user and performs the operations with less execution time and less energy the MCC provides all the resources to the mobile user which they are connected through the internet there are further techniques developing in the cloud for task offloading with less execution time and consuming less energy the technique used by the authors in this paper is to offload the task of the mobile users to the cloud servers based on parameters such as offloading scope, partitioning and migration[3].
The cloud exploits a new era of computing. In this type of computing applies services are benefited with the help of Internet. cloud computing can extends the capacity of computing for mobiles, but this is not the final solution for extending the systems batteries lifetime. We can get the final solution for the systems battery extensions[8]. The mobile users can save energy by performing operations over the internet. the numerical results of this paper significantly show that the mobile devices can save there energy by offloading mobile applications to the cloud remotely[7]. The technique DVFS is used to reduce the power consumption. The scheduling of task in mobile cloud computing is essential for performing the operations efficiently the scheduling algorithm allocate the task execution time[11].

\section{PROPOSED SYSTEM}

mobile cloud computing provide so many resources for the mobile user using so many techniques as a user offloads multiple task to the cloud and face so many problems like more energy consumption, more cpu utilization and time taking. As we seen in the existing system the files are offloaded in a mobile manager and after long time they are send to cloud manager for execution this process utilize much amount of time and uses more energy .To overcome this we developed a new technique parallelization. By parallelization the mobile manager communicates to the cloud manager in multiple path and also execute the file in multipath.

by using the commands of the data owner the data is forwarded to another user by storage servers. The system model consists of splitting the uploaded file into many chunks and transferring the files to cloud using various routing paths with encrypted data. the storage system allocates different data containers to the user data. By using AES encryption mechanism the user uploads the data to the servers. The system secures the data by 
segregation process.in cloud storage the data is stored in different locations and will be saved in pieces. when the another user send a request for the data the cloud system will provide the data by using reversible manner. Hence our system protects the data from attackers. In this we have designed transferring of files to cloud server using multipath framework technique file is divide into multiple blocks each block is transmitted in multiple network routes using multiple routes transmission it reduces file transmission time. total network paths can be used they were will not get any network jams efficiency of transmission will increase.

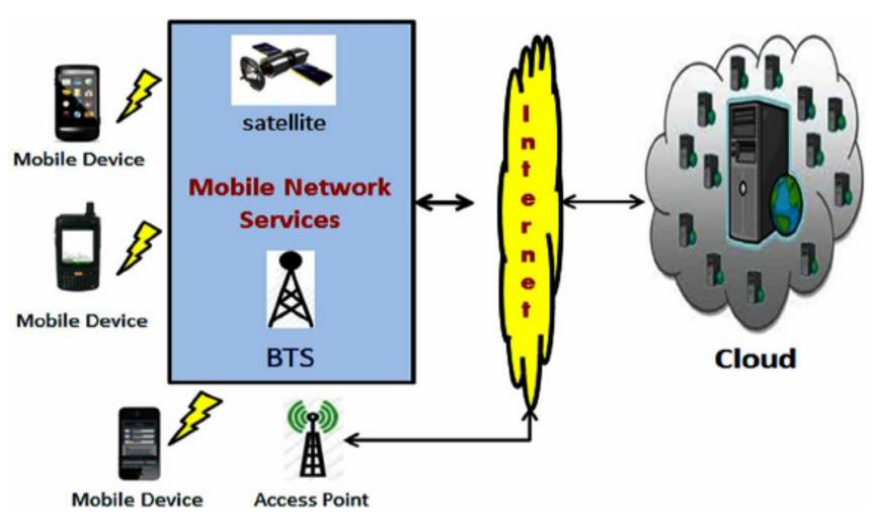

Figure 2 : Proposed System Architecture

We use parallel data transmission for mobiles, tabs and other wireless devices they can transmit files faster. Here various wireless devices are connected to service provider connected various cloud getting various packets for multiple route. To make faster upload/download and save cpu utilization time and network transmission time. Every mobile user should register in the cloud, for every user a unique id is generated.by using this id the user can upload the file, then the file splits into many chunks a secret key is generated for every uploaded file.by using this secret key the user can download the file where the splits gets merged and downloaded.

\section{Algorithm}

Input: CPU utilization, input size, usage of memory and energy consumption.

Output: result time for each method, transmission of files.

1: Read annotated method name.

2: Check network status and signal strength by using Monitor Module.

3: if there is no connection

4: Execute locally on mobile devices.

5: else

6: For each method 'I' do

7: Read Transfer, CPU, memory, and power through profile module.

8. if the decision is offloading then

9: Encrypts the data method by using the AES Algorithm.

10: Send to the cloud for remote execution.

11: Return (Result back to the mobile device).

12: else

13: Execute locally on the mobile devices.

14. end if

15: end for

16: end if

17: Update the profile file with new values.

18.Display network paths used, time consumed, cpu utilized. End

The system model consists of splitting the uploaded file into many chunks and transferring the files to cloud using various routing paths with encrypted data. the mobile devices uploads the data with AES encryption mechanism, the are transfer to cloud server through the cloud manager in cloud servers the data pieces are stored in different locations. When a client needs the data, the cloud server should provides the data in reversible manner. 


\section{Advantages}

By using the parallelization concept we can offload the task on considering the constraints like time of execution, CPU utilization, memory and energy consumption. By using AES encryption technique it provide security to the data by encryption.

\section{EXPEREMENTAL RESULTS}

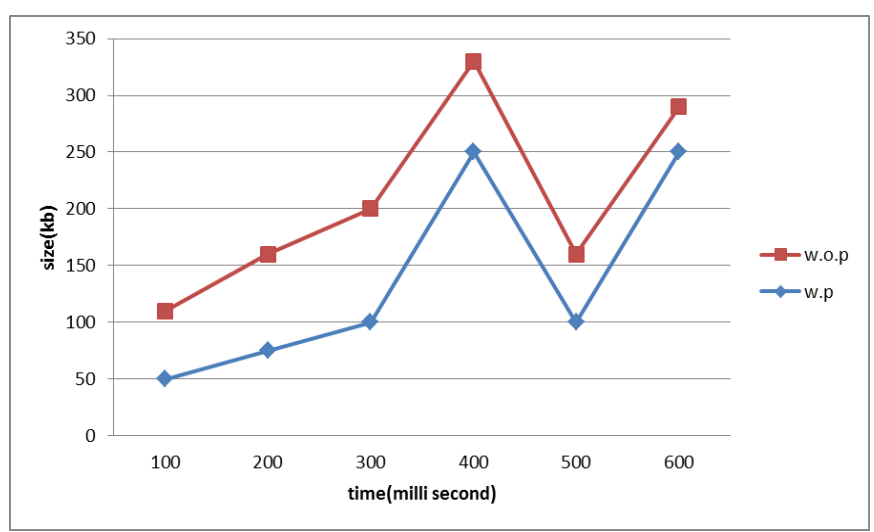

In the above figure we notice the execution time for the task off loader to the cloud the cloud performs the task by taking large time and utilizing more energy. In proposed system we use a technique parallelization to reduce the both execution time and energy consumption by observing the graph we can come to now how parallelization technique reducing the execution time for every task given comparing to the existing system.

\section{CONCLUSION}

As now a days the mobile users are facing so many problems to overcome them we develop a few techniques one among them is optimization by using this optimization in cloud computing we can reduce energy consumption, cpu utilization, memory usage so that the users can do the task with in less time and perform there task with great efficiency. In future work we can use this technique in multi tasking and perform a number of tasks in a same time with providing a security

\section{REFERENCES}

[1]. S. Kosta, A. Aucinas, P. Hui, R. Mortier, and X. Zhang, "ThinkAir: Dynamic resource allocation and parallel execution in the cloud for mobile code offloading," in Proc. IEEE 31st Annu. Int. Conf. Comput. Commun. (INFOCOM), 2012, pp. $945-953$.

[2]. R. Kemp, N. Palmer, T. Kielmann, and H. Bal, "Cuckoo: A computation offloading framework for smartphones," in Proc. 2nd Int. ICST Conf. Mobile Comput., Appl. Services, 2012, pp. 5979.

[3]. M. Shiraz, A. Gani, R. H. Khokhar, and R. Buyya, "A review on distributed application processing frameworks in smart mobile devices for mobile cloud computing," IEEE Communications Surveys \& Tutorials, vol. 15, no. 3, pp. 1294-1313, 2013.

[4]. Ibrahim A Elgendy, Wei-Zhe Zhang, Member, IEEE, Chuan-Yi Liu, and Ching-Hsien Hsu, senior Member, IEEE An Efficient and Secured Framework for Mobile Cloud Computing

[5]. M. Burton and D. Felker, Android Application Development for Dummies. For Dummies, 2012.

[6]. S. Guo, B. Xiao, and Y. Yang, "Energy-efficient dynamic offloading and resource scheduling in mobile cloud computing", IEEE INFOCOM, pp. 1-9, 2016.

[7]. W. Zhang, Y. Wen, K. Guan, K. Dan, H. Luo, and $\mathrm{D}$. O. $\mathrm{Wu}$, "Energyoptimal mobile cloud computing under stochastic wireless channel," IEEE Transactions on Wireless Communications, vol. 12, no. 9, pp. 4569-4581, 2013.

[8]. K. Kumar and Y.-H. Lu, "Cloud computing for mobile users: Can offloading computation save energy?" Computer, vol. 43, no. 4, pp. 51-56, Apr. 2010.

[9]. M. Nir, A. Matrawy, and M. St-Hilaire, "An energy optimizing scheduler for mobile cloud 
computing environments," in Proc. IEEE Conf. Comput. Commun. Workshops, 2014, pp. 404409

[10]. I. A. Elgendy, M. El-Kawkagy, and A. Keshk, "An efficient framework to improve the performance of mobile applications," International Journal of Digital Content Technology \& Its Applications, vol. 9, no. 5, pp. 43-54, 2015.

[11]. Lin, Y. Wang, Q. Xie, and M. Pedram, "Task scheduling with dynamic voltage and frequency scaling for energy minimization in the mobile cloud computing environment," IEEE Transactions on Services Computing, vol. 8, no. 2, pp. 175-186, 2015.

[12]. B. Aggarwal, P. Chitnis, A. Dey, K. Jain, V. Navda, V. N. Padmanabhan, R. Ramjee, A. Schulman, and N. Spring, "Stratus: Energyefficient mobile communication using cloud support," ACM SIGCOMM Comput. Commun. Rev., vol. 41, no. 4, pp. 477-478, 2011.

\section{Cite this article as :}

N. Divya, "Secured Cloud Resources Scheme Using Multi-Process Technique", International Journal of Scientific Research in Computer Science, Engineering and Information Technology (IJSRCSEIT), ISSN : 2456-3307, Volume 7 Issue 1, pp. 155-160, JanuaryFebruary 2021. Available at doi : https://doi.org/10.32628/CSEIT217132 Journal URL : http://ijsrcseit.com/CSEIT217132 